\title{
Electromagnetic bronchoscopy guided microwave ablation for early stage lung cancer presenting as ground glass nodule
}

\author{
Feichao Bao", Fenghao Yu\#, Rui Wang, Chunji Chen, Yonghui Zhang, Boyu Lin, Yiyang Wang, \\ Xiuxiu Hao, Zhitao Gu, Wentao Fang
}

Department of Thoracic Surgery, Shanghai Chest Hospital, Shanghai Jiao Tong University, Shanghai, China

Contributions: (I) Conception and design: F Bao, F Yu, R Wang, Z Gu, W Fang; (II) Administrative support: R Wang, C Chen, Y Zhang, B Lin, Y Wang, X Hao; (III) Provision of study materials or patients: F Bao, F Yu, R Wang, C Chen, Y Zhang; (IV) Collection and assembly of data: C Chen, Y Zhang, B Lin, Y Wang, X Hao; (V) Data analysis and interpretation: F Bao, F Yu; (VI) Manuscript writing: All authors; (VII) Final approval of manuscript: All authors.

"These authors contributed equally to this work.

Correspondence to: Wentao Fang; Zhitao Gu. Department of Thoracic Surgery, Shanghai Chest Hospital, Shanghai Jiao Tong University, NO. 241, West Huaihai Road, Shanghai 200000, China. Email: vwtfang@hotmail.com; guzhitao0717@hotmail.com.

Background: Patients with early-stage lung cancer are sometimes medically inoperable, and for patients with multiple primary lung cancers, surgical resection alone sometimes proves to be impractical. Local treatments like microwave ablation (MWA) are investigational alternatives for these patients. Most reported MWA procedures for lung cancers are performed percutaneously under CT guidance. MWA navigated by electromagnetic bronchoscopy (ENB) has been limitedly studied. In this study, we aimed to evaluate the safety and feasibility of MWA under ENB guidance in patients with inoperable early-stage lung cancers or multiple primary lung cancers which cannot be completely resected.

Methods: From June 2019 to December 2020, preliminary attempts of ENB-guided MWA were made in five medically inoperable patients with a single early-stage lung cancer and ten patients with multiple primary lung cancers which were difficult to resect at the same time. For patients with concomitant pulmonary nodules which needed surgical resection, thoracoscopic resections were performed following ENB-guided MWA. The safety, feasibility, and technique effectiveness of treatments were evaluated.

Results: ENB-guided MWA for 15 ground glass nodules (GGNs) in 15 patients was completed in accordance with the planned protocol. Biopsy of 13 GGNs showed malignancy. Five patients received simple ENB-guided MWA without simultaneous surgical resection and ten patients received simultaneous surgical resection for 13 concomitant pulmonary nodules. CT scan by the first postoperative week showed technique effectiveness of ablation for 11 nodules indicated for MWA. Four patients had mild complications after the procedure and recovered shortly after treatment.

Conclusions: For medically inoperable patients with a single GGN manifesting early-stage lung cancer and patients with multiple primary early-stage lung cancers which cannot be resected at the same time, ENB-guided MWA might be a safe and feasible alternative local treatment, whether combined with surgical resection or not. However, large, prospective, randomized, multicenter studies are needed to confirm its role in the treatment of early-stage lung cancer.

Keywords: Lung cancer; ground glass nodule (GGN); electromagnetic bronchoscopy (ENB); microwave ablation (MWA); video-assisted thoracoscopic surgery (VATS)

Submitted Jun 07, 2021. Accepted for publication Aug 23, 2021.

doi: $10.21037 /$ tlcr-21-474

View this article at: https://dx.doi.org/10.21037/tlcr-21-474 


\section{Introduction}

Lung cancer is the leading cause of cancer-related death worldwide, and for patients with operable diseases, surgical resection is the best option (1). With the widespread use of computed tomography (CT), early detection of lung cancer is made possible and managing patients with lung cancers presenting as ground glass nodules (GGN) has become routine for thoracic surgeons. However, approximately $5-25 \%$ of early-stage lung cancers cannot be removed surgically (2-4), and for patients with multiple GGNs, the situation is more complicated. Surgical resection alone sometimes proves to be impractical because complete resection of all nodules might require multiple complex segmentectomies, bilobectomy or even bilateral surgery or pneumonectomy, which would result in increased introoperative risks and postoperative morbidity, as well as decreased postoperative life quality due to cardiopulmonary dysfunction and other complications. Non-surgical ablative procedures are thus investigated as therapeutic alternatives in early-stage lung cancer for patients not suitable for surgery $(5,6)$. For treatment of GGN manifesting lung cancers, because they are air-rich lesions, RFA requires saline injection to decrease the impedance between its applicator and targeted lesions, while direct ablation without saline injection is feasible for MWA. Most reported MWA for lung cancer are performed percutaneously under CT guidance $(5,6)$; however, CT-guided ablation has limitations. Due to its invasive nature, higher intraoperative and postoperative risks are expected for patients with poor pulmonary function and centrally located lesions, and for central targets with long distance to pleural surface a certain level of technical difficulty also remains (7). Electromagnetic bronchoscopy (ENB) which could display images of the bronchial tree provides a less invasive and more versatile solution: it plans and generates potential pathways for navigation using CT images, and gains a more natural access to targeted lesions via bronchial tree for biopsy and treatment. ENB has been used for biopsy of peripheral pulmonary lesions with relatively high diagnostic yield especially for lesions with the bronchus sign $(8,9)$. Up to now, all reported MWA treatments for early-stage lung cancer have been performed almost exclusively percutaneously, except for a limited number of reported cases that combined ENB-guided MWA with surgical resection for treatment of multiple primary lung cancers (10-12). In this study we aimed to evaluate the feasibility, safety and technique effectiveness of ENB-guided MWA for patients with GGN manifesting lung cancers who underwent the procedure with or without surgical resection for concomitant pulmonary lesions. We present our article in accordance with the STROBE reporting checklist (available at https://dx.doi.org/10.21037/tlcr-21-474).

\section{Methods}

\section{Study population}

The study was conducted in accordance with the Declaration of Helsinki (as revised in 2013). The study was approved by institutional review board of Shanghai Chest Hospital (NO. XS2021) and informed consent was taken from all the patients. For the purposes of this study, preliminary attempts were conducted on patients with GGN manifesting lung cancer. A multidisciplinary team including thoracic surgeons, radiologists and medical oncologists carefully evaluated patients' medical operability and technical achievability of successful ablation before granting the procedure. Between June 2019 and December 2020, ENBguided MWA was accomplished in five medically inoperable patients with a single early-stage lung cancer and in ten patients with multiple primary lung cancers which were difficult to resect at the same time. Indications for ENBguided MWA included: (I) patients not suitable for surgical resection due to poor cardiopulmonary function and other comorbidities; (II) patients with operable lesions refusing surgical treatment; (III) patients with synchronous multiple primary tumors, resections of which might require bilateral surgery (Figure 1), lobectomy plus segmentectomy (Figure 2), multiple lobectomies (Figure 3), or even pneumonectomy. Exclusion criteria included: (I) lesions easily reached by percutaneous ablation (located in the outer third of the lung, neither near the mediastinal pleura nor covered by anatomic barrier such as scapula or ribs); (II) lesions without suitable pathway after meticulous pathway planning; (III) patients with coagulation disorder, active inflammation or infection. Comorbidity here is defined as the co-existence of disorders other than lung cancer in the same individual, for example, cardiovascular disease, other types of concomitant cancers and so on. The following data were collected for analysis: patient and tumor characteristics, procedure characteristics including navigation and MWA parameters, complications and local efficacy.

\section{Procedure}

All ENB procedures used the superDimension ${ }^{\mathrm{TM}}$ navigation 

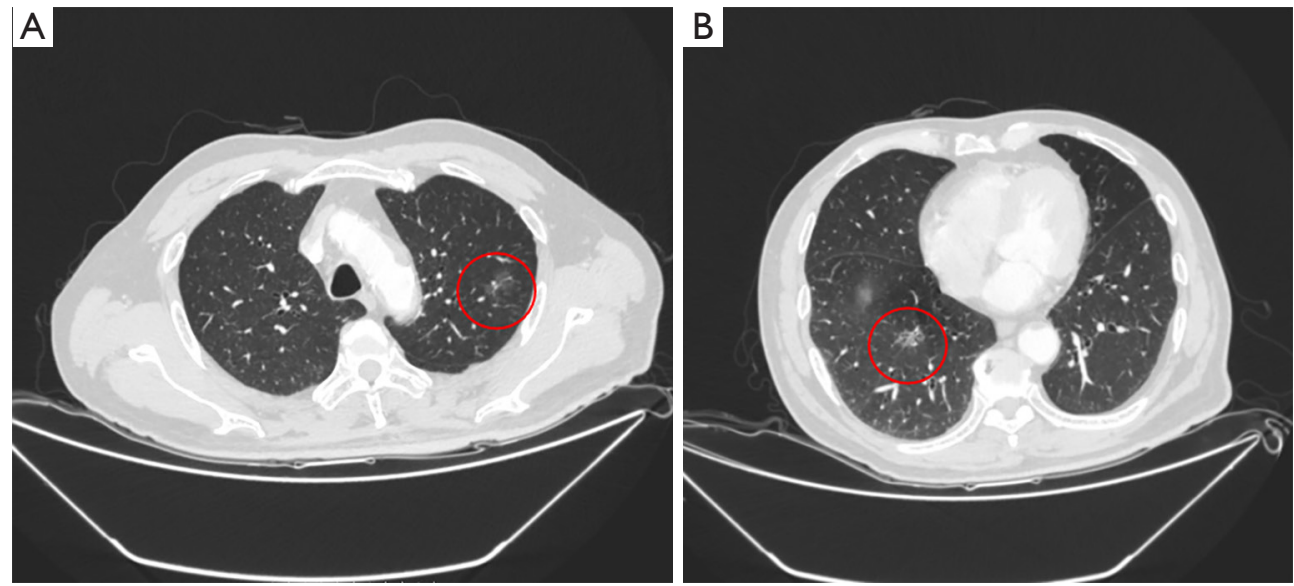

Figure 1 A 75-year-old male smoker with two nodules. (A) A 1.8- cm mGGN in left upper lobe (marked with red circle), was resected by left upper trisegmentectomy; (B) a 1.7- cm mGGN located in the central right upper lobe (marked with red circle), which required lobectomy for resection, was treated by ENB-guided MWA. mGGN, mixed ground glass nodule; ENB, electromagnetic bronchoscopy; MWA, microwave ablation.
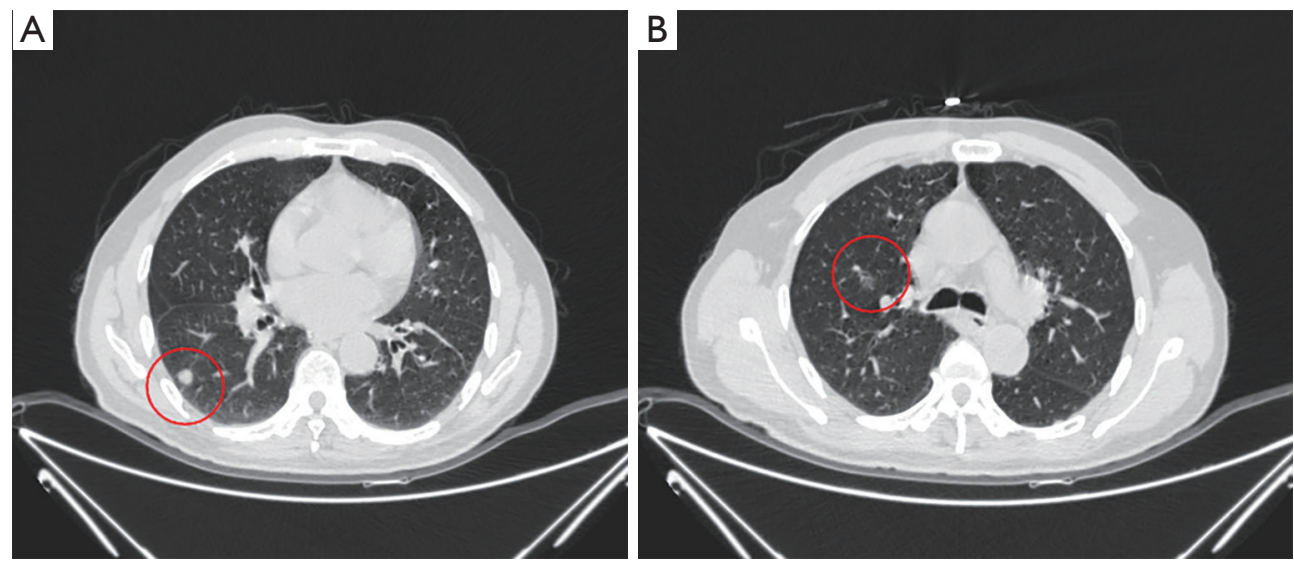

Figure 2 A 65-year-old male smoker with two nodules. (A) A 1.2- cm solitary pulmonary nodule in right lower lobe (marked with red circle) was resected by right superior segmentectomy via VATS; (B) a 0.9- $\mathrm{cm}$ mGGN located in the central right upper lobe (marked with red circle), which required lobectomy for resection, was treated by ENB-guided MWA. VATS, video-assisted thoracoscopic surgery; mGGN, mixed ground glass nodule; ENB, electromagnetic bronchoscopy; MWA, microwave ablation.

system version 7.0 (Medtronic, Minneapolis, USA). The system consists of four basic elements: an electromagnetic field; an Edge ${ }^{\mathrm{TM}}$ steerable and locatable guide; an Edge ${ }^{\mathrm{TM}}$ extended working channel with different angles and procedure planning software. A flexible bronchoscope with a 2.8-mm working channel was used for ENB procedure (Pentax Inc; Tokyo, Japan). An MWA device was used for ablation (Nanjing Visionmedic, Nanjing, China). CT scan for planning was performed after hyperventilation using a multidetector scanner which qualified the requirement of procedure planning software. Patients were examined in the supine position and scanned contiguously for the chest cavity with the use of intravenous contrast. One-mm-thick or 0.63 -mm-thick sections were reconstructed following the soft tissue algorithm. After pathway planning was confirmed using the procedure-planning software, patients were put under monitored anesthesia care with midazolam and fentanyl and intubated with laryngeal mask throughout the ENB procedure. Following the completion of anesthesia, ENB then proceeded: the extended working channel was docked onto the bronchoscope and the locatable guide was introduced through the working channel; the tip of the 

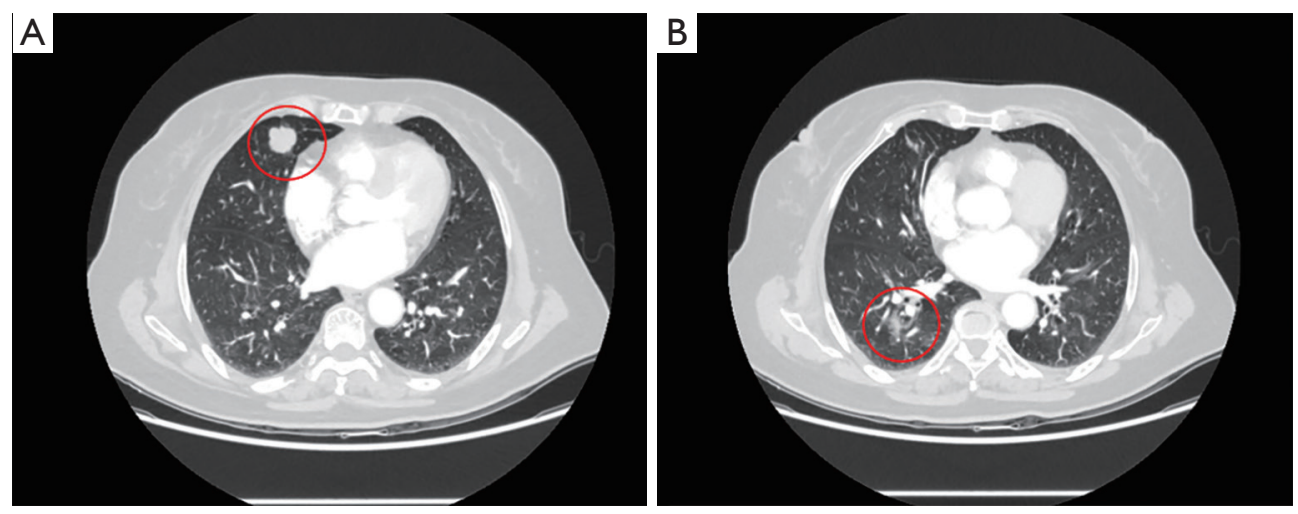

Figure 3 A 72-year-old female with two nodules (A) A 2.1- cm solitary pulmonary nodule in right middle lobe (marked with red circle) was resected by right middle lobectomy via VATS; (B) a 1.7- cm mGGN (marked with red circle) located in the central right lower lobe, which required lobectomy for resection, was treated by ENB-guided MWA. VATS, video-assisted thoracoscopic surgery; mGGN, mixed ground glass nodule; ENB, electromagnetic bronchoscopy; MWA, microwave ablation.

locatable guide probed inside airways under navigation till it reached the target lesion; thereafter, the locatable guide was retrieved and replaced by tissue biopsy tools; tissue samples were obtained by aspiration needles, biopsy forceps, and cytology brushes and then assessed with rapid onsite evaluation (ROSE). Ablation was carried out immediately after malignancy was confirmed by ROSE. For nodules which ROSE showed no sign of malignancy, MWA was still given with patients' informed consent prior to the procedure because the nodules were radiologically suspicious on a series of follow-up CT scans. The antenna of MWA was introduced via the extended working channel to the lesion and connected to the microwave generator. Power was often set as $40-80 \mathrm{~W}$ and duration time ranged from 5 to 10 minutes, depending on the size and shape of the targeted lesion. An antenna track ablation was performed following lesion ablation to avoid tumor implantation. If the patient had other concomitant pulmonary nodules which required further surgical resection, general anesthesia was further achieved by propofol and fentanyl. They were then intubated with a double-lumen endotracheal tube to establish singlelung ventilation during surgery and extubated at the end of the operation. Video assisted thoracoscopic surgery (VATS) was performed to resect concomitant lesions by lobectomy, segmentectomy or wedge resection based on the location and invasiveness of the lesion.

\section{Follow-up evaluation}

MWA effectiveness was evaluated by CT scan within the first week after treatment. The ablation zone was the radiological region of the post-ablation change characterized by a solid or ground-glass opacity area around the tumor $(13,14)$. According to standardization of terminology and reporting criteria for image-guided tumor ablation $(15,16)$, "technique effectiveness" refers to a "complete ablation" of macroscopic tumor as evidenced by the imaging of a tumor covered by a larger solid or ground-glass opacity area (Figure 4). While technical success is defined as that MWA antenna is placed at the planned sites of each tumor and ablation is completed according to the planned protocol. It should be noted that a major challenge of ablation has been the lack of reliable postprocedural assessment of the response to treatment. No standard imaging protocol for post-ablation follow-up has been established. Postprocedural imaging is only a rough guide to the success of ablation therapy, since microscopic foci of residual tumor is impossible to identify on imaging and can only be confirmed by re-biopsy (17).

\section{Statistical analysis}

Continuous variables are presented as the mean values \pm standard deviations. Categorical variables are presented as numbers and frequencies (\%). All statistical analyses were performed using Microsoft Excel 2016 (Microsoft Corporation, WA, USA).

\section{Results}

From June 2019 to December 2020, fifteen consecutive 

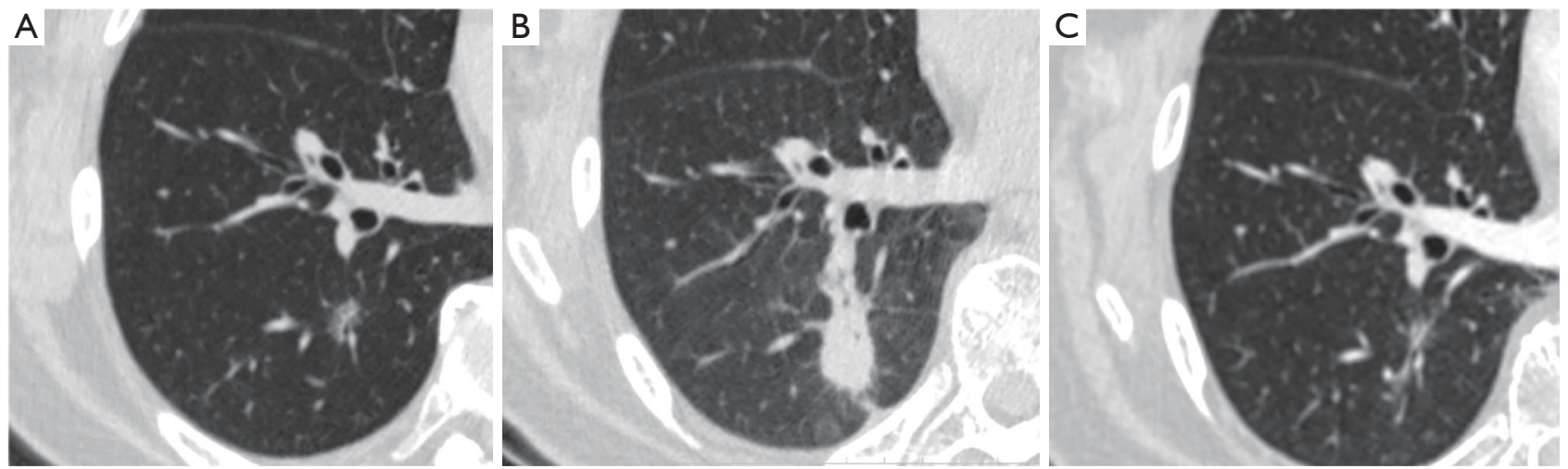

Figure 4 Representative images of CT scans. (A) A 50-year-old female patient with a 1.1- cm mGGN on right lower lobe prior to MWA; (B) tumor lesion seen on CT on the first postoperative day: the ablation zone encompassed the lesion with a margin; (C) CT scan at 24 weeks after ENB-guided MWA: the ablation zone was replaced by parenchymal changes different from the original nodule. However, further follow-up CT scans are needed to confirm the effectiveness of ablation. CT, computed tomography; mGGN, mixed ground glass nodule; MWA, microwave ablation; ENB, electromagnetic bronchoscopy.

patients with GGN manifesting lung cancer underwent ENB-guided MWA (Table 1). Indications for ENB-guided MWA included multiple lesions ( $\mathrm{N}=10)$, poor pulmonary function due to previous thoracic surgeries $(\mathrm{N}=4)$, and refusal to receive surgical resection $(\mathrm{N}=1)$. All lesions indicated for ENB-guided MWA were no more than $3 \mathrm{~cm}$ and were air-rich lesions (GGN). Such lesions are more suitable for MWA. All 15 nodules for ENB-guided MWA were considered not suitable for percutaneous ablation due to various reasons. Thirteen of them were located in the inner two thirds of the lung, therefore distant from pleura. Two were located in the outer third of the lung but still deemed not suitable for percutaneous ablation: one nodule was at the mediastinal side of the lung; for the other nodule the patient himself had previous contralateral lobectomy and percutaneous ablation was considered to be potentially fatal if the intact lung should collapse. Five patients received ENB-guided MWA without simultaneous surgical resection, while the other ten patients underwent surgical resection immediately after ablation. Together, all 13 concomitant pulmonary nodules in ten patients were resected through VATS: 6 by wedge resection, 4 by segmentectomy and 3 by lobectomy (Table 2). Out of the ten patients who received the hybrid procedure of ENB-guided MWA and thoracoscopic resection, 2 patients received combined treatment for nodules in contralateral lungs, 7 received treatment for nodules in ipsilateral lungs but different lobes, and a 73-year-old female patient received combined treatment for nodules in the same lobe (Figure 5)-a left lower lobe lobectomy was deemed intolerable for this patient due to compromised pulmonary function after previous right upper lobe lobectomy and right mastectomy. Therefore, the patient received ENB-guided MWA for the centrally located nodule, and a wedge resection for the peripheral nodule. All of the surgical resections were done smoothly after ENBguided MWA. According to IASLC's Staging Manual (18), tumors should be considered multifocal ground glass lung adenocarcinoma if there are multiple sub-solid nodules (either pure ground glass or part-solid), with at least one suspected or proven to be cancer. And this applies whether or not a biopsy has been performed. All nodules in our study were either pGGN or sub-solid therefore met the criteria so nodules undergoing MWA represented synchronous primary as those resected.

ENB-guided MWA for all 15 GGNs was completed in accordance with the planned treatment protocol. The technical success rate was $100 \%$. Biopsy was obtained and ROSE was performed for all 15 nodules before MWA was carried out and all biopsied tissues were further examined by routine histology examination. For 13 nodules, ablation was offered immediately after biopsied tissues were proved to be malignant by ROSE. For two nodules which ROSE showed no signs of malignancy, MWA was still given with patients' informed consent prior to the procedure because the nodules were radiologically suspicious on a series of follow-up CT scans. Four of the 15 patients had mild complications including air leak, pneumonia and hemoptysis after procedure, with two of them considered 
Table 1 Demographic characteristics of patient and tumor treated for MWA

\begin{tabular}{|c|c|}
\hline Variable & Cases $(\mathrm{N})$ \\
\hline Age (mean \pm SD) & $58.7 \pm 15.7$ \\
\hline \multicolumn{2}{|l|}{ Gender } \\
\hline Female & 10 \\
\hline Male & 5 \\
\hline \multicolumn{2}{|l|}{ Comorbidity } \\
\hline Yes & 7 \\
\hline None & 8 \\
\hline \multicolumn{2}{|l|}{ Size(cm) } \\
\hline$\leq 1$ & 8 \\
\hline$>1$ but $\leq 2$ & 4 \\
\hline$>2$ but $\leq 3$ & 3 \\
\hline \multicolumn{2}{|l|}{ Density } \\
\hline pGGN & 2 \\
\hline mGGN & 13 \\
\hline \multicolumn{2}{|l|}{ Bronchus sign } \\
\hline Yes & 10 \\
\hline None & 5 \\
\hline \multicolumn{2}{|l|}{ Contour } \\
\hline Smooth & 11 \\
\hline Spiculated & 4 \\
\hline \multicolumn{2}{|l|}{ Tumor location } \\
\hline RUL & 7 \\
\hline RML & 2 \\
\hline RLL & 4 \\
\hline LUL & 1 \\
\hline LLL & 1 \\
\hline
\end{tabular}

MWA, microwave ablation; RUL, right upper lobe; RML, right middle lobe; RLL, right lower lobe; LUL, left upper lobe; LLL, left lower lobe; pGGN, pure ground glass nodule; mGGN, mixed ground glass nodule.

not related to MWA but to surgical resection and the other two patients with hemoptysis regarded as MWA induced; hemoptysis of both patients was noticed on postoperative day one and they recovered swiftly after intravenous antibiotics and coagulation treatment. No pneumothorax or massive bleeding occurred in this case series (Table 3). All
15 patients were discharged on the 1st to 8 th postoperative day. CT scans by the first postoperative week showed technique effectiveness for ablation in 11 nodules (73.3\%) (Table 4). Larger energy output and longer duration of MWA treatment were used for three lesions of larger size $(2-3 \mathrm{~cm})$. Post-ablation images showed that technique effectiveness was achieved in two of them.

\section{Discussion}

Surgical resection remains the standard of care for patients of early-stage lung cancer, with a 5 -year overall survival higher than $90 \%$ after surgery (19). But for various reasons such as cardiopulmonary dysfunction and other comorbidities, about $5-25 \%$ of early-stage lung cancers cannot be treated by surgery (2-4). Meanwhile, multiple pulmonary lesions located in different lobes are being increasingly detected (20). Decision making for this group of patients usually puts clinicians in a dilemma, especially for those with bilateral pulmonary lesions. Various surgical approaches including synchronous bilateral VATS and subxiphoid VATS have been explored as solutions for their effectiveness but are associated with higher risks $(21,22)$ : surgical resection of sizable lung parenchyma is difficult to tolerate, thus increasing morbidity and mortality. Ablation treatments have been investigated as alternative options for patients unsuitable for surgery $(5,6)$.

Both RFA and MWA use thermal energy to cause coagulative necrosis of tumor tissue. RFA has been more widely used and studied for the local treatment of lung cancer. MWA is a promising, relatively new ablation technique: several studies have suggested that MWA is effective for both primary and metastatic tumors, both early and advanced stage lung cancer $(6,23,24)$. Compared with RFA, MWA generates higher temperature in a shorter period of time and is less subject to high impedance from high temperature and heat sink effect, thus producing larger and more homogeneous ablation zone which is dependent on both the power output and duration of MWA treatment (20,21). Moreover, direct ablation without saline injection between applicator tip and target is feasible for MWA when it comes to air-rich lesions like GGN manifesting lung cancer. Therefore, MWA offers advantages and shows superiority in the treatment of GGN manifesting lung cancer.

Percutaneous MWA under CT guidance has been the mainstream technique for peripheral pulmonary nodules, but due to high technical difficulty or intra-operative and post-operative risks patients with certain nodules 
Table 2 Characteristics of patients with multiple nodules received combined treatment

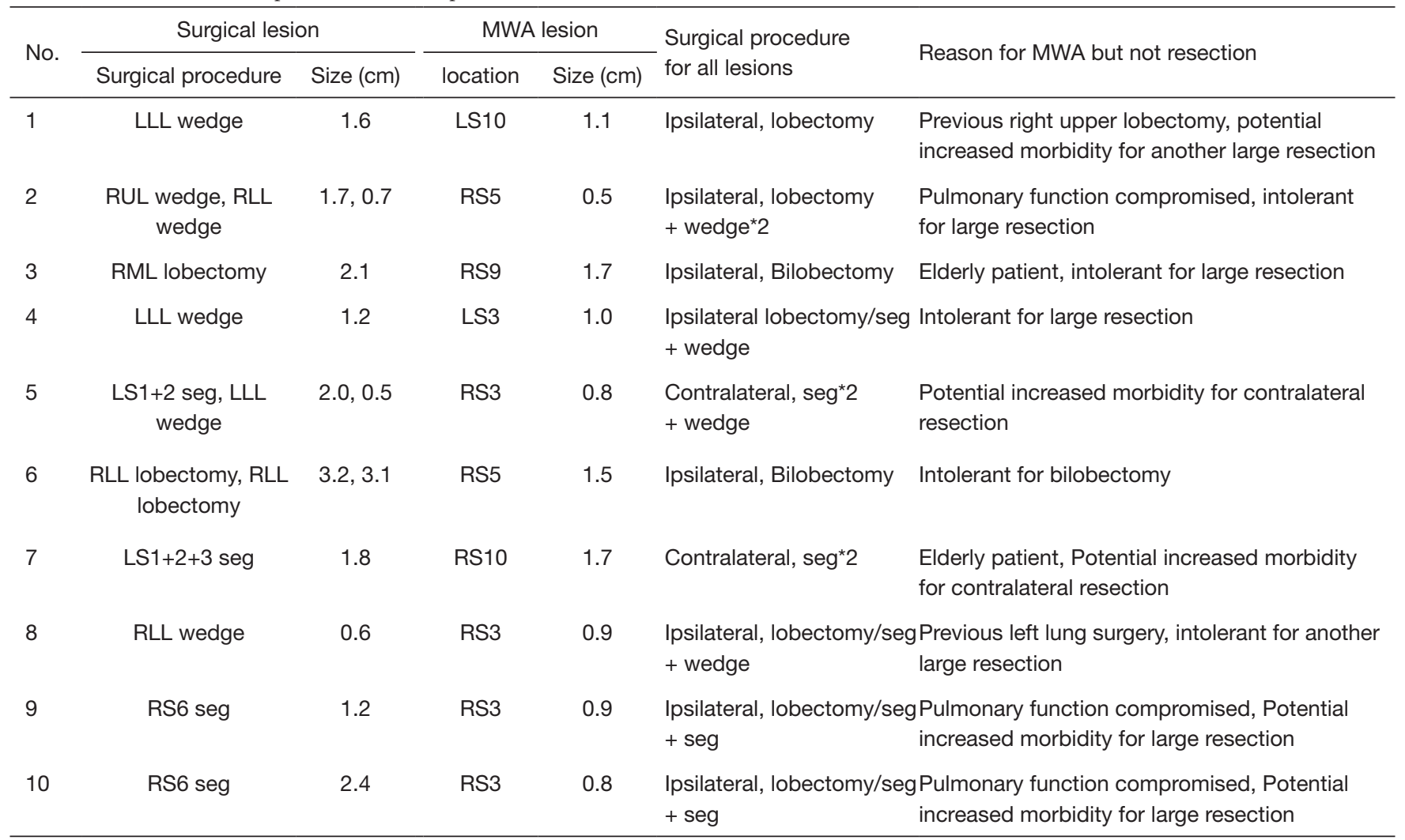

MWA, microwave ablation; Seg, segmentectomy; RUL, right upper lobe; RML, right middle lobe; RLL, right lower lobe; LLL, left lower lobe.

are deemed unsuitable for percutaneous MWA. Nodules covered by anatomic barriers such as the scapula or a rib, nodules distant to the pleural surface are difficult to target under CT guidance, and for small nodules distant to the pleura surface, the risk of pneumothorax would dramatically increase (25). Patients with severe emphysema are at high risk of persistent air leak requiring chest drainage. Another downside is that patients would suffer from severe pain for ablation of lesions near the pleura (7). ENB is recommended for biopsy and treatment of peripheral lesions, but theoretically it could reach all targets in the lung. Compared with percutaneous CT-guided biopsy, ENB-guided biopsy has comparable diagnostic accuracy and lower incidence of complications for smaller and deeper lesions (26). However, reports on ENB-guided MWA were limited (27). Up to now, all reported microwave ablations for early-stage lung cancer were performed percutaneously except for limited cases (10-12). The patient in one case report had bilateral pulmonary lesions: the lesion in the right upper lobe was not suitable for wedge resection and was treated by ENBguided MWA, followed by thoracoscopic wedge resections of three lesions in the left hemithorax (10). Another report with 11 cases combined ENB-guided microwave ablation with VATS resection for multiple GGNs; however, the ablation effectiveness was limitedly stated (11).

In our study, all nodules indicated for ablation were reached in accordance with the planned protocol. The high technical success rate was first owed to strict patient selection. Lesions of larger sizes or with bronchus signs would be easier to reach successfully $(9,28,29)$. In this study, biopsy results came back positive for malignancy for all three nodules sized $2-3 \mathrm{~cm}$. Ten nodules for ENB showed the bronchus sign on CT scan, and were much easier for navigation; while the other 5 nodules were at the extension of terminal bronchus and did not show bronchus sign but were reached nonetheless without the use of any transbronchial tool. The second reason for high technical success rate of navigation was detailed preoperative path planning: we would abandon ENB-guided MWA if the lesion in concern was difficult to reach under navigation.

Ablation for most nodules (11/15) indicated for ENBguided MWA in this study was considered to be technically 

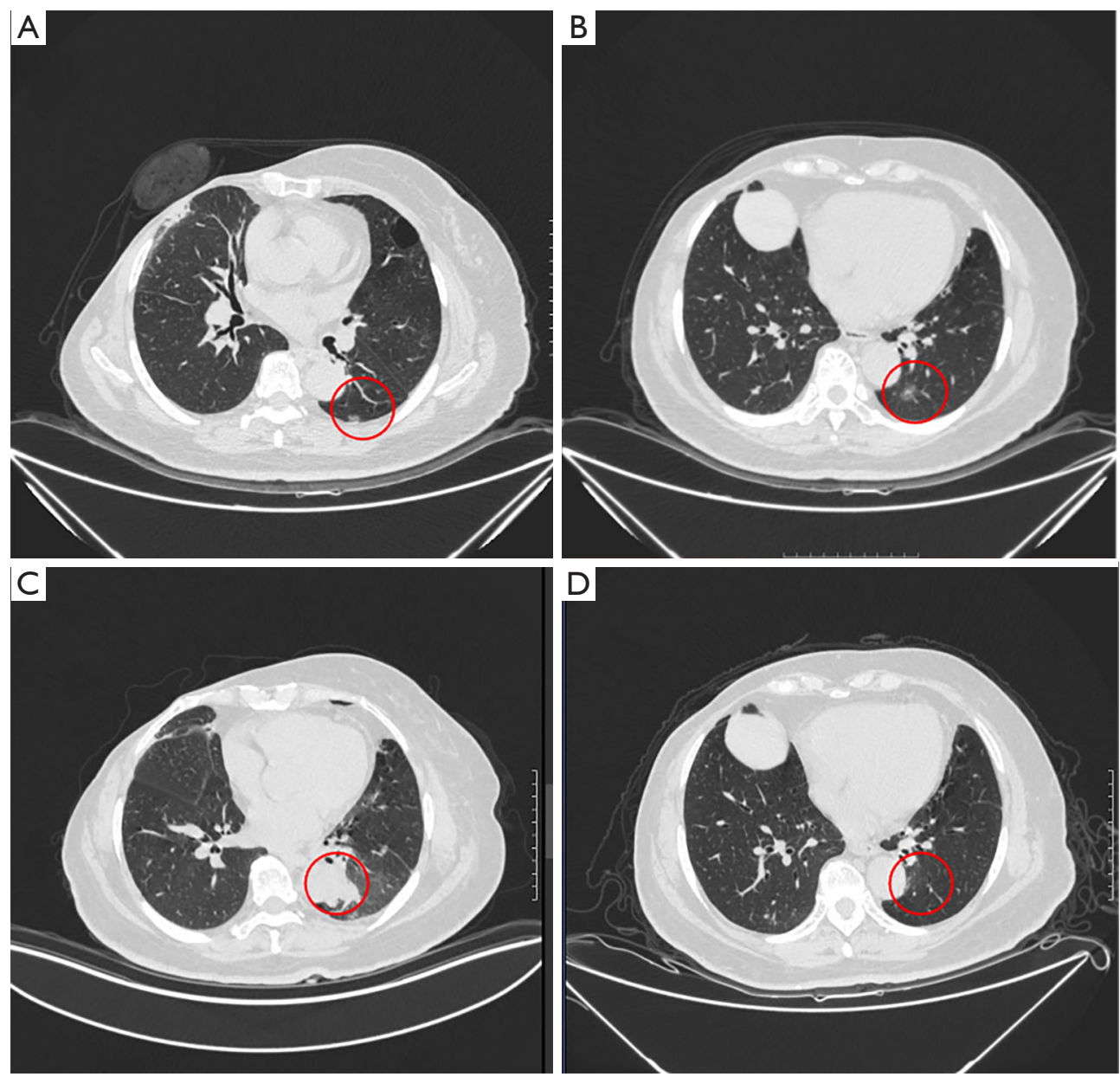

Figure 5 A 73 -year-old female patient with previous history of right upper lobectomy and right mastectomy received combine treatment for two nodules in the same lobe. (A) The peripherally located nodule (marked with red circle) was resected by VATS wedge resection; (B) the mGGN (marked with red circle), which seemed not suitable for wedge resection or simple segmentectomy, was treated by MWA; (C) tumor lesion seen on CT on the first postoperative day: the ablation zone (marked with red circle) encompassed the MWA lesion with a margin; (D) CT scan at 24 weeks after ENB-guided MWA: the primary location of the lesion (marked with red circle) was replaced by parenchyma bands. mGGN, mixed ground glass nodule; MWA, microwave ablation; CT, computed tomography; ENB, electromagnetic bronchoscopy.

Table 3 Data of patients with complication after ENB guided MWA

\begin{tabular}{lllll}
\hline Patient characteristics & Complication & ENB procedure & Surgical procedure & Management \\
\hline 57 yo, male & Air leak & RS5 mGGN MWA & RLL lobectomy & Drainage \\
67 yo, male & Hemoptysis & RS1 mGGN MWA & None & Hemostatic agents \\
75 yo, male & Hemoptysis & RS10 mGGN MWA & LS1+2+3 segmentectomy & Hemostatic agents \\
65 yo, male & Pulmonary infection & RS3 mGGN MWA & RS6 segmentectomy & Antibiotics \\
\hline
\end{tabular}

ENB, electromagnetic bronchoscopy; MWA, microwave ablation; mGGN, mixed ground glass nodule; RLL, right lower lobe; yo, years old. 
Table 4 Procedure characteristics of ENB guided MWA

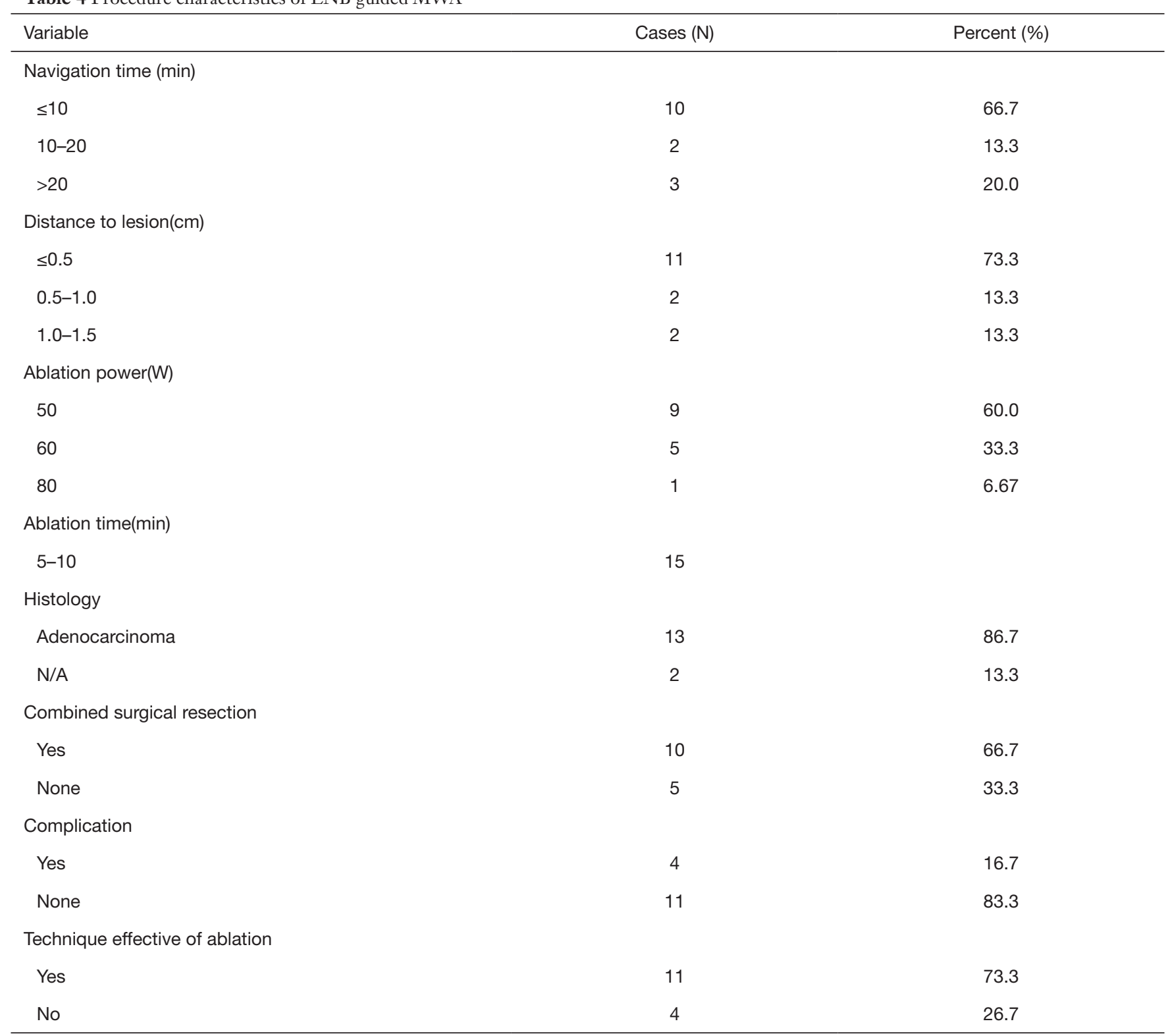

N/A, no available proof of histology of malignancy was obtained. ENB, electromagnetic bronchoscopy; MWA, microwave ablation.

effective. Based on our preliminary experience and published literatures, we proposed several tips that would potentially improve the technique effectiveness of ENBguided MWA. The first tip is the use of transbronchial access tool like CrossCountry ${ }^{\mathrm{TM}}$, which was designed to gain access to pulmonary nodules outside an airway $(30,31)$, as more than $50 \%$ of nodules are located outside a visible airway. The use of transbronchial access tool would make ENB-guided MWA technically more suitable for more lesions even without bronchial pathway. The second is about the location sensor, as the location sensor of superDimension $^{\mathrm{TM}}$ is placed within the tip of the locatable guide which probes inside the airways, and after the target is located the locatable guide is then replaced by sampling instruments or ablation antenna without any real-time image guidance. The following biopsy and ablation are in a way performed 'blind', which may lower the diagnostic yield and lead to technical failure of ablation. A continuous tracking system which embeds the electromagnetic sensors in the tip of the sampling instruments and ablation antenna 
would make the procedure more precise. The third improvement would be the use of cone beam CT (CBCT) imaging to confirm the antenna location $(9,31)$, but the experience on CBCT for detecting GGN is limited, and the technique still needs to be improved.

\section{Limitation}

This study had some limitations. Firstly, it was a nonrandomized, single-arm study with a small patient pool and it lacked intermediate and long-term follow up data. Nevertheless, the preliminary results supported the utility of ENB-guided MWA as feasible with low incidence of complications for early-stage lung cancer. Secondly, due to high false-negative rate of biopsy for GGNs (28), two patients underwent ablation without definitive malignant histology; their final diagnoses were made based on lesion increase confirmed by serial followup CT scans supporting high clinical probability of primary malignancy, rather than on pathology. Thirdly, no reliable postprocedural assessment of the response to treatment has been established, meanwhile, the long-term oncologic effect has not been collected. Despite limitations, these encouraging results provided a useful framework for future prospective studies to compare ENB-guided MWA with other alternative treatment for early-stage lung cancer.

\section{Conclusions}

For medically inoperable patients with simple GGN manifesting early stage lung cancer, and patients with multiple GGN manifesting primary lung cancers which cannot be resected at the same time, ENB-guided MWA could be a potentially safe, feasible and technically effective alternative for local treatment whether in combination or not with surgical resection, but large prospective randomized multicenter studies are still needed to further confirm its role in the management of early-stage lung cancer.

\section{Acknowledgments}

Funding: The study was partly funded by National Natural Science Foundation of China (Grant Award Number 81900099).

\section{Footnote}

Reporting Checklist: The authors have completed the
STROBE reporting checklist. Available at https://dx.doi. org/10.21037/tlcr-21-474

Data Sharing Statement: Available at https://dx.doi. org/10.21037/tlcr-21-474

Peer Review File: Available at https://dx.doi.org/10.21037/ tlcr-21-474

Conflicts of Interest: All authors have completed the ICMJE uniform disclosure form (available at https://dx.doi. org/10.21037/tlcr-21-474). The authors have no conflicts of interest to declare.

Ethical Statement: The authors are accountable for all aspects of the work in ensuring that questions related to the accuracy or integrity of any part of the work are appropriately investigated and resolved. The study was conducted in accordance with the Declaration of Helsinki (as revised in 2013). The study was approved by institutional review board of Shanghai Chest Hospital (NO. XS2021) and informed consent was taken from all the patients.

Open Access Statement: This is an Open Access article distributed in accordance with the Creative Commons Attribution-NonCommercial-NoDerivs 4.0 International License (CC BY-NC-ND 4.0), which permits the noncommercial replication and distribution of the article with the strict proviso that no changes or edits are made and the original work is properly cited (including links to both the formal publication through the relevant DOI and the license). See: https://creativecommons.org/licenses/by-nc-nd/4.0/.

\section{References}

1. Siegel RL, Miller KD, Jemal A. Cancer statistics, 2019. CA Cancer J Clin 2019;69:7-34.

2. Puri V, Crabtree TD, Bell JM, et al. Treatment Outcomes in Stage I Lung Cancer: A Comparison of Surgery and Stereotactic Body Radiation Therapy. J Thorac Oncol 2015;10:1776-84.

3. Bryant AK, Mundt RC, Sandhu AP, et al. Stereotactic Body Radiation Therapy Versus Surgery for Early Lung Cancer Among US Veterans. Ann Thorac Surg 2018;105:425-31.

4. Lee K, Kim HO, Choi HK, et al. Real-world treatment patterns for patients 80 years and older with early lung cancer: a nationwide claims study. BMC Pulm Med 2018;18:127. 
5. Vogl TJ, Nour-Eldin NA, Albrecht MH, et al. Thermal Ablation of Lung Tumors: Focus on Microwave Ablation. Rofo 2017;189:828-43.

6. Moussa AM, Ziv E, Solomon SB, et al. Microwave Ablation in Primary Lung Malignancies. Semin Intervent Radiol 2019;36:326-33.

7. Bhatt KM, Tandon YK, Graham R, et al. Electromagnetic Navigational Bronchoscopy versus CT-guided Percutaneous Sampling of Peripheral Indeterminate Pulmonary Nodules: A Cohort Study. Radiology 2018;286:1052-61.

8. Munoz-Largacha JA, Litle VR, Fernando HC. Navigation bronchoscopy for diagnosis and small nodule location. J Thorac Dis 2017;9:S98-103.

9. Folch EE, Pritchett MA, Nead MA, et al. Electromagnetic Navigation Bronchoscopy for Peripheral Pulmonary Lesions: One-Year Results of the Prospective, Multicenter NAVIGATE Study. J Thorac Oncol 2019;14:445-58.

10. Jiang N, Zhang L, Hao Y, et al. Combination of electromagnetic navigation bronchoscopy-guided microwave ablation and thoracoscopic resection: An alternative for treatment of multiple pulmonary nodules. Thorac Cancer 2020;11:1728-33.

11. Qu R, Tu D, Hu S, et al. ENB-guided microwave ablation combined with uniportal VATS for multiple ground glass opacities. Ann Thorac Surg 2021. [Epub ahead of print]. doi: 10.1016/j.athoracsur.2021.04.061.

12. Chan JWY, Lau RWH, Ngai JCL, et al. Transbronchial microwave ablation of lung nodules with electromagnetic navigation bronchoscopy guidance-a novel technique and initial experience with 30 cases. Transl Lung Cancer Res 2021;10:1608-22.

13. Araujo-Filho JAB, Menezes R, Horvat N, et al. Lung radiofrequency ablation: post-procedure imaging patterns and late follow-up. Eur J Radiol Open 2020;7:100276.

14. Abtin FG, Eradat J, Gutierrez AJ, et al. Radiofrequency ablation of lung tumors: imaging features of the postablation zone. Radiographics 2012;32:947-69.

15. Dupuy DE, Zagoria RJ, Akerley W, et al. Percutaneous radiofrequency ablation of malignancies in the lung. AJR Am J Roentgenol 2000;174:57-9.

16. Goldberg SN, Grassi CJ, Cardella JF, et al. Image-guided tumor ablation: standardization of terminology and reporting criteria. J Vasc Interv Radiol 2009;20:S377-90.

17. Steinfort DP, Christie M, Antippa P, et al. Bronchoscopic Thermal Vapour Ablation for Localized Cancer Lesions of the Lung: A Clinical Feasibility Treat-and-Resect Study. Respiration 2021;100:432-42.
18. Detterbeck FC, Nicholson AG, Franklin WA, et al. The IASLC Lung Cancer Staging Project: Summary of Proposals for Revisions of the Classification of Lung Cancers with Multiple Pulmonary Sites of Involvement in the Forthcoming Eighth Edition of the TNM Classification. J Thorac Oncol 2016;11:639-50.

19. Hattori A, Takamochi K, Oh S, et al. Prognostic Classification of Multiple Primary Lung Cancers Based on a Ground-Glass Opacity Component. Ann Thorac Surg 2020;109:420-7.

20. Ghosh S, Mehta AC, Abuquyyas S, et al. Primary lung neoplasms presenting as multiple synchronous lung nodules. Eur Respir Rev 2020;29:190142.

21. Giraldo Ospina CF, Mongil Poce R, Arrabal Sánchez R, et al. Subxiphoid uniportal video-assisted bilateral surgery: right upper lobectomy and left upper wedge resection S3. J Vis Surg 2017;3:186.

22. Cai H, Xie D, Al Sawalhi S, et al. Subxiphoid versus intercostal uniportal video-assisted thoracoscopic surgery for bilateral lung resections: a single-institution experience. Eur J Cardiothorac Surg 2020;57:343-9.

23. Zheng A, Ye X, Yang X, et al. Local Efficacy and Survival after Microwave Ablation of Lung Tumors: A Retrospective Study in 183 Patients. J Vasc Interv Radiol 2016;27:1806-14.

24. Pusceddu C, Melis L, Sotgia B, et al. Usefulness of percutaneous microwave ablation for large non-small cell lung cancer: A preliminary report. Oncol Lett 2019;18:659-66.

25. Yeow KM, Su IH, Pan KT, et al. Risk factors of pneumothorax and bleeding: multivariate analysis of 660 CT-guided coaxial cutting needle lung biopsies. Chest 2004;126:748-54.

26. Xie F, Zheng X, Xiao B, et al. Navigation BronchoscopyGuided Radiofrequency Ablation for Nonsurgical Peripheral Pulmonary Tumors. Respiration 2017;94:293-8.

27. Lau K, Spiers A, Pritchett M, et al. P1.05-06 Bronchoscopic Image-Guided Microwave Ablation of Peripheral Lung Tumours - Early Results. J Thorac Oncol 2018;13:S542.

28. Seijo LM. Electromagnetic navigation bronchoscopy: clinical utility in the diagnosis of lung cancer. Lung Cancer (Auckl) 2016;7:111-8.

29. Sato T, Yutaka Y, Ueda Y, et al. Diagnostic yield of electromagnetic navigational bronchoscopy: results of initial 35 cases in a Japanese institute. J Thorac Dis 2018;10:S1615-9.

30. Ishiwata T, Gregor A, Inage T, et al. Bronchoscopic 
navigation and tissue diagnosis. Gen Thorac Cardiovasc Surg 2020;68:672-8.

31. Bowling MR, Brown C, Anciano CJ. Feasibility and
Safety of the Transbronchial Access Tool for Peripheral Pulmonary Nodule and Mass. Ann Thorac Surg 2017;104:443-9.

Cite this article as: Bao F, Yu F, Wang R, Chen C, Zhang Y, Lin B, Wang Y, Hao X, Gu Z, Fang W. Electromagnetic bronchoscopy guided microwave ablation for early stage lung cancer presenting as ground glass nodule. Transl Lung Cancer Res 2021;10(9):3759-3770. doi: 10.21037/tlcr-21-474 